\title{
Hyphomycetes isolados da água e do solo da Reserva Florestal de Dois Irmãos, Recife, PE, Brasil
}

\author{
Marilene da Silva Cavalcanti ${ }^{1,3}$ e Adauto Ivo Milanez ${ }^{2}$
}

Recebido em 12/01/2006. Aceito em 5/03/2007

\begin{abstract}
RESUMO - (Hyphomycetes isolados da água e do solo, da Reserva Florestal de Dois Irmãos, Recife, PE, Brasil). Visando ampliar o conhecimento sobre a diversidade de fungos em ambientes aquáticos, coletas da água e do solo das margens dos açudes do Vale do Prata e do Meio foram efetuadas na Reserva Florestal de Dois Irmãos, Recife, Estado de Pernambuco. Dentre outras, foram isoladas cinco espécies pouco comuns de Hyphomycetes. As amostras de água foram coletadas abaixo da lâmina d'água e as de solo nas margens dos referidos açudes. As amostras de solo foram submetidas a diluições até 1:10000 e $1 \mathrm{~mL}$ de cada suspensão foi plaqueado no meio de Martin acrescido de cloranfenicol $(50 \mathrm{mg} / \mathrm{L})$. Alíquotas $(1 \mathrm{~mL})$ das amostras de água foram semeadas no mesmo meio. As placas foram deixadas em temperatura ambiente $\left(27^{\circ} \mathrm{C} \pm 2\right)$, durante 3-4 dias, para o desenvolvimento de colônias e posterior isolamento dos fungos. Dentre as espécies identificadas Curvularia tuberculata Jain, Dendrosporium lobatum Plakidas \& Edgerton ex Crane, Dichotomophthoropsis nymphaearum (Rand) M. B. Ellis, Phaeoisaria glauca (Ellis \& Everh.) Hoog \& Papendorf e Trichurus spiralis Hasselbring são destacadas porque haviam sido pouco referidas e não descritas anteriormente no Brasil.
\end{abstract}

Palavras-chave: diversidade, fungos, taxonomia

\begin{abstract}
Hyphomycetes from water and soil at the Dois Irmãos Forest Reserve, Recife, Pernambuco State, Brazil). To increase our knowledge regarding fungal diversity in aquatic environments, water and soil were collected at the edge of two dams (Açude do Vale do Prata and Açude do Meio) at the Dois Irmãos Forest Reserve, Recife, Pernambuco state. Five uncommon species of Hyphomycetes were found, among others. Water samples were collected below the water surface and soil samples from the shores of these dams. Soil samples were diluted to 1:10000, and $1 \mathrm{~mL}$ of each suspension was plated in Martin's medium with $50 \mathrm{mg} / \mathrm{L}$ chloranphenicol. Water samples $(1 \mathrm{~mL})$ were plated in the same medium. The plates were left at room temperature $\left(27{ }^{\circ} \mathrm{C} \pm 2\right)$ for $3-4$ days, for colony development and subsequent species isolation. Among the identified species, Curvularia tuberculata Jain, Dendrosporium lobatum Plakidas \& Edgerton ex Crane, Dichotomophthoropsis nymphaearum (Rand) M.B. Ellis, Phaeoisaria glauca (Ellis \& Everh.) Hoog \& Papendorf and Trichurus spiralis Hasselbring have rarely been cited and have yet not been described in Brazil.
\end{abstract}

Key words: diversity, fungi, taxonomy

\section{Introdução}

Os Hyphomycetes desempenham importante papel nos ecossistemas, atuando como sapróbios ou parasitas. São encontrados tanto nos ambientes terrestres quanto aquáticos. Grande parte desses fungos tem o solo como seu principal reservatório, contribuindo para a reciclagem de nutrientes e fertilidade do mesmo. Como sapróbios, são responsáveis por boa parte da decomposição da matéria orgânica, atuando nas transformações bioquímicas nos ecossistemas, uma vez que a atividade de degradação libera compostos mais simples, os quais são reabsorvidos pelas plantas (Remacle 1981). Esses fungos também aumentam a palatabilidade dos substratos para consumo dos detritívoros, dinamizando a reciclagem de nutrientes ou servindo de alimento por meio da produção de biomassa (Christensen 1989; Kendrick 1992; Alexopoulos et al. 1996; MooreLandecker 1996). Outros são importantes na cadeia trófica pela habilidade que têm de degradar a lignina, sobreviver em condições anaeróbicas, reproduzir-se em águas marinhas e resistir a concentrações elevadas de metais pesados (Field \& Webster 1983; Fisher et al. 1983; Abel \& Barlocher 1984; Sridhar \& Kaveriappa 1989; Raghukumar 2000; Watanabe et al. 2000).

\footnotetext{
1 Universidade Federal de Pernambuco, Departamento de Micologia, Laboratório de Fungos Aquáticos, CCB, Cidade Universitária, 50670-420 Recife, PE, Brasil

2 Instituto de Botânica, Seção de Micologia e Liquenologia, C. Postal 3005, 01061-970 São Paulo, SP, Brasil

3 Autora para correspondência: silva.cordeiro@globo.com
} 
858 Cavalcanti \& Milanez: Hyphomycetes isolados da água e do solo, da Reserva Florestal de Dois Irmãos, Recife, PE...

A Reserva Florestal de Dois Irmãos, onde estão localizados os açudes do Prata e do Meio, é um dos remanescentes da Mata Atlântica no Estado de Pernambuco e está situada na região Noroeste da cidade de Recife $\left(8^{\circ} 04^{\prime} 00^{\prime \prime} \mathrm{S}\right.$ e $\left.34^{\circ} 52^{\prime} 00^{\prime \prime} \mathrm{W}\right)$, encravada junto a áreas densamente populosas. Estes açudes, originados de poços artesianos, afloramentos de lençóis subterrâneos, precipitação pluviométrica e de águas provenientes das encostas, são de propriedade da COMPESA (Companhia Pernambucana de Saneamento), utilizados para o abastecimento de água de parte da cidade do Recife e desempenham importante papel no ecossistema, por estarem localizados no interior de uma Reserva de Mata Atlântica. Estudos de cunho ecológico e de biodiversidade visando o conhecimento, recuperação e preservação da Reserva de Dois Irmãos vêm sendo desenvolvidos (Machado et al. 1998). Pesquisas sobre a diversidade de fungos filamentosos em áreas de Mata Atlântica ainda são escassas, apesar das contribuições para os Estados de São Paulo, por Grandi \& Attili (1996); Gusmão \& Grandi (1997); Grandi (1998; 1999); Schoenlein-Crusius \& Milanez (1998a; 1998b); Wellbaum et al. (1999); Grandi \& Gusmão (2002) dentre outras, e de Pernambuco, por Maia $(1983 ; 1998)$ e Maia et al. (2002). No entanto, ainda faltam dados sobre a distribuição geográfica, as interações ecológicas e a diversidade dos fungos nesse bioma.

O objetivo desse trabalho foi descrever cinco espécies de Hyphomycetes, dentre as 250 isoladas na área de estudo. Essas espécies foram citadas apenas em listagens de fungos (Maia 1998; Maia et al. 2002), com base em registros da Micoteca URM, sendo apresentada agora a descrição desses táxons, considerando o caráter pouco comum de sua ocorrência no Brasil.

\section{Material e métodos}

Foram coletadas, trimestralmente, amostras de água e de solo das margens dos açudes do Vale do Prata e do Meio, na Reserva Florestal de Dois Irmãos, Recife, Pernambuco, no período de janeiro/1996 a dezembro/1997. Para as amostragens foram escolhidas cinco estações de coleta, sendo uma de cada lado (norte, sul, leste e oeste) e uma no centro de cada manancial. No total foram coletadas 40 amostras de água no açude do Prata e 40 no açude do Meio. As amostras de água foram coletadas abaixo da lâmina d'água, em recipientes de boca larga, previamente esterilizados. As amostras de solo foram coletadas nas margens dos açudes com auxílio de uma espátula esterilizada, e acondicionadas em sacos plásticos. No laboratório, $1 \mathrm{~mL}$ de cada amostra de água foi semeado em placas de Petri contendo meio de Martin (1950), com o pH ajustado para 6,5, adicionado de antibiótico (cloranfenicol $50 \mathrm{mg} / \mathrm{L}$ ). Para as amostras de solo foi utilizada a técnica de Warcup (1967) modificada, de maneira a permitir uma suspensão final de partículas de solo de 1:10.000. Em seguida, $1 \mathrm{~mL}$ de cada suspensão foi plaqueado no mesmo meio. As placas foram deixadas em temperatura ambiente $\left(27^{\circ} \mathrm{C} \pm 2\right)$ e após 3-4 dias de incubação, as colônias foram transferidas para placas de Petri contendo meio batatadextrose-ágar (BDA) ou "corn-meal-agar" (CMA), até a identificação das espécies.

A identificação das espécies foi baseada em características microestruturais e análise comparativa de parâmetros estabelecidos na taxonomia convencional, de acordo com literatura especializada. Amostras das espécies identificadas foram depositadas na Micoteca URM (Departamento de Micologia, Universidade Federal de Pernambuco).

\section{Resultados e discussão}

Curvularia tuberculata Jain, Trans. Br. Mycol. Soc. 45: 539, 1962.

Fig. 1-2

Colônia em BDA com crescimento rápido, atingindo toda a placa de Petri aos sete dias de cultivo, cotonosa, castanho-escura a castanho-clara no centro, margem regular, reverso liso, negro; conidióforos laterais ou terminais, eretos ou flexuosos, simples ou ramificados, castanho-escuros, 50-200×2-7 $\mu$ m; células conidiogênicas terminais ou intercalares, simpodiais, levemente intumescida, com cicatrizes; conídios solitários, retos, fusiformes ou elipsoidais, parede tuberculada, usualmente 3 septos transversais, castanho-pálidos a castanho-escuros, células das extremidades claras, 25-49×10,5-16 $\mu \mathrm{m}$.

Material examinado: BRASIL. Pernambuco: Recife, XII/1996, III/1997, VI/1997, água e solo, Cavalcanti s.n. (Micoteca URM-3957).

Distribuição geográfica: Índia, Iraque, Tanzânia e Indochina (Ellis 1971a); Taiwan (Matsushima 1980; 1983). Brasil: Pernambuco (Maia 1998; Maia et al. 2002) e Ceará (Freire 2005; Freire \& Kozakiewicz 2005).

Comentários: as características apresentadas pelos espécimes são similares às descritas por Jain 
(1962) e Ellis (1971b). Curvularia tuberculata foi isolada pela primeira vez de folhas de Zea mays L. e grãos de Oryza sativa L. na Índia (Jain 1962). Ellis (1971a) refere o isolamento de C. tuberculata de Pinus sp., do ar atmosférico, do solo e de material têxtil na Índia, Indochina, Iraque e Tanzânia. Sivanesan (1985) descreve o estágio teleomorfo (Cochliobolus tuberculatus Sivan.) por emparelhamento monoconidial de espécies heterotálicas compatíveis.

Dendrosporium lobatum Plakidas \& Edgerton ex Crane, Trans. Br. Mycol. Soc. 58: 423, 1972.

Fig. 3-4

Colônia em CMA, aos sete dias de cultivo, com cerca de $6 \mathrm{~cm}$ diâm; cotonosa, castanho-oliváceoescura, zonada, margem fimbriada; reverso liso, castanho-escuro; conidióforos simples ou ramificados, flexuosos ou retos, simples ou ramificados, hialinos, terminais ou intercalares com desenvolvimento simpodial, 9,0-14,5×2,8-3,6 $\mu$ m; células conidiogênicas terminais, proliferando simpodialmente; conídios hialinos, achatados, triangulares, constritos lateralmente, usualmente com três lóbulos de cada lado, 1-septado, sendo o lóbulo basal mais largo, pedicelado, 10,8-14,5×4,5-7,2 $\mu \mathrm{m}$.

Material examinado: BRASIL. Pernambuco: Recife, III/1996 e VI/1997, solo, Cavalcanti s.n. (Micoteca URM-3953).

Distribuição geográfica: Estados Unidos da América (Plakidas \& Edgerton 1936); Índia (Agnihothrudu 1963); Venezuela (Castañeda-Ruiz et al. 2003); México (Rivera 2003) e Brasil (Maia 1998; Maia et al. 2002).

Comentários: as características apresentadas pelos espécimes concordaram com as descrições de Plakidas \& Edgerton (1936); Agnihothrudu (1963) e Crane (1972). Dendrosporium foi descrito em 1936 por Plakidas \& Edgerton, tendo como espécie-tipo $D$. lobatum, isolado de cascas de pêra (Pyrus serotina Rehd.) em decomposição, em Louisiana (EUA) e cultivado em meios de vagem de feijão-ágar, dextroseágar e Czapek-ágar. Entretanto, os autores não apresentaram a diagnose latina da espécie, o que foi feito por Crane (1972), que isolou D. lobatum de frutos em decomposição de Fuglans nigra L.

Dichotomophthoropsis nymphaearum (Rand) M. B. Ellis, Mycol. Pap. 125: 22, 1971.

Fig. 5-6

Colônia em BDA com crescimento rápido, atingindo toda a placa de Petri aos sete dias de cultivo, cotonosa a pulverulenta, pardacenta, tornando-se rosa no centro, margem irregular; reverso negro. $\mathrm{Na}$ maturidade, a colônia apresenta pontuações caracterizando os esclerócios e presença abundante de exsudato. Conidióforos macronematosos ou semimacronematosos, ramificados, freqüentemente dicotômicos ou tricotômicos, septados, subhialinos a castanho-claros, lisos; células conidiogênicas lobadas, politréticas, 21,5-130×3,6-4,5 $\mu \mathrm{m}$; conídios solitários em cada lóbulo, cilíndricos, curvos, helicoidais, multiseptados, equinulados, castanho-claros, 75,8-277,5x 6,5-7,2 $\mu \mathrm{m}$; esclerócios subesféricos, castanho-escuros a negros, 74,1-66,7×81,4-74,1 $\mu \mathrm{m}$.

Material examinado: BRASIL. Pernambuco: Recife, II/1996, III/1996, VI/1996, IX/1996, XII/1996, III/1997, VI/1997, IX/1997, água e solo, Cavalcanti s.n. (Micoteca URM-3954, 3955, 3956).

Distribuição geográfica: Índia (Wright et al. 1990), Estados Unidos da América (Ellis 1971a; Hoog \& Guarro 1995) e Brasil (Maia 1998; Maia et al. 2002).

Comentários: as características apresentadas pelos espécimes concordaram com as descrições de Ellis (1971b) e Hoog \& Guarro (1995). Dichotomophthoropsis foi proposto por Ellis (1971b) para fungos isolados de folhas de Nymphaea odorata Aiton, N. pubescens Willd e N. stellata Willd na Índia e Estados Unidos da América. Hoog \& Guarro (1995) descreveram a espécie mencionando a presença de conídios mais largos $(6-20 \mu \mathrm{m})$. Helicosporium nymphaearum Rand, Gyroceras nymphaearum (Rand) Linder e Helicoceras nymphaearum (Rand) Linder foram considerados por Ellis (1971a) sinônimos de Dichotomophthoropsis nymphaearum. Mazzanti Gutiérrez (1995) isolaram Helicoceras orysae Linder $\&$ Tullis de sementes de arroz, na Argentina, e sugeriram que o táxon deveria ser incluído em D. nymphaearum. Wright et al. (1990) mencionaram a espécie, na Índia, causando queratomicose no homem.

Phaeoisaria glauca (Ellis \& Everh.) de Hoog \& Papendorf, Persoonia 8: 414, 1976.

Fig. 7-8

Colônia em BDA com crescimento lento, atingindo $4,0 \mathrm{~cm}$ diâm. aos sete dias de cultivo, inicialmente apresentando coloração castanho-clara, tornando-se posteriormente acinzentada; reverso castanho, exsudato ausente; sinemas castanho-claros, 130-319x 101-116 $\mu \mathrm{m}$; células conidiogênicas simpodiais originadas de hifas não diferenciadas, com 11-15x 2,8-3,6 $\mu \mathrm{m}$ larg. na base; conídios sobre dentículos, unicelulares, lisos, hialinos, gutulados, elipsoidais, parede 
860 Cavalcanti \& Milanez: Hyphomycetes isolados da água e do solo, da Reserva Florestal de Dois Irmãos, Recife, PE...

fina, base pontiaguda, 3,6-4,5×1,8-2,5 $\mu \mathrm{m}$.

Material examinado: BRASIL. Pernambuco: Recife, III/1996, VI/1996, IX/1996, XII/1996, XI/1997, XII/1997, água, Cavalcanti s.n. (Micoteca URM-4004).

Distribuição geográfica: cosmopolita (Domsch et al. 1993).
Comentários: as características apresentadas pelos espécimes concordam com as descrições de Hoog \& Papendorf (1976) e de Domsch et al. (1993). Phaeoisaria glauca era conhecida como Chloridium glaucum Ellis \& Everh (seu basiônimo), que foi isolado de madeira em decomposição (Quercus sp.) nos Estados Unidos da América. O táxon foi isolado por

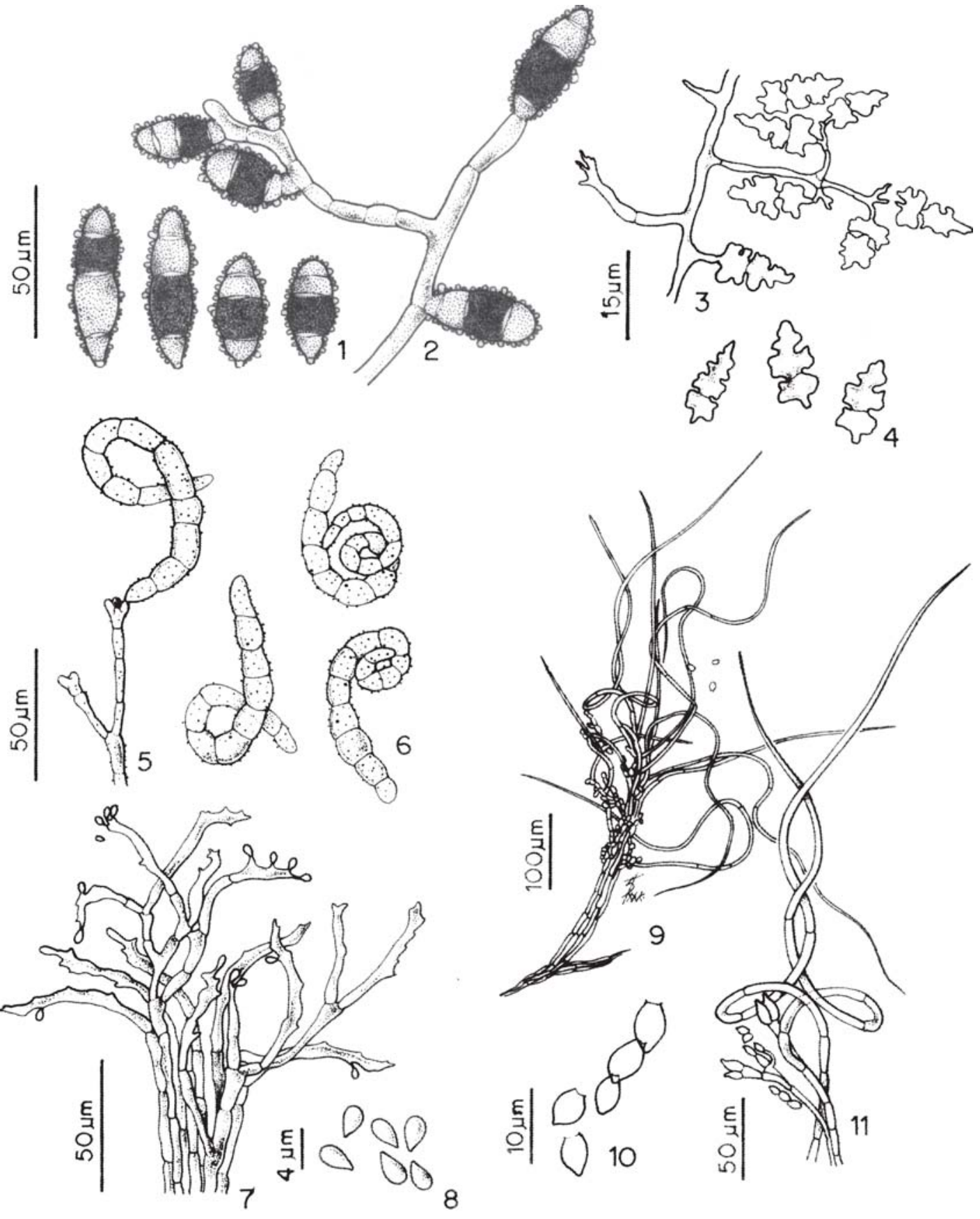

Figuras 1-11. 1-2. Curvularia tuberculata Jain. 1. Conídios. 2. Conidióforos e conídios. 3-4. Dendrosporium lobatum Plakidas \& Edgerton ex Crane. 3. Conidióforos e conídios. 4. Conídios. 5-6. Dichotomophthoropsis nymphaearum (Rand) M.B. Ellis. 5. Conidióforos. 6. Conídios. 7-8. Phaeoisaria glauca (Ellis \& Everh.) de Hoog \& Papendorf. 7. Aspecto parcial do sinema com células conidiogênicas. 8. Conídios. 9-11. Trichurus spiralis Hasselbring. 9. Aspecto geral do sinema. 10. Conídios. 11. Células conidiogênicas e setas. 
Domsch et al. (1993) em Acer sp. (Holanda) e em Buxus sempervirens L. (França); Hoog \& Papendorf (1976) isolaram P. glauca de madeira em decomposição, na Rússia. Grandi \& Attili (1996) citaram duas espécies de Phaeoisaria para o Brasil: $P$. clematidis (Fckl.) Hughes e $P$. infrafertilis Sutton \& Hodges, isoladas de folhas de Alchornea triplinervia (Spreng.) M. Arg. na Reserva Ecológica de Juréia-Itatins, em São Paulo, SP.

Trichurus spiralis Hasselbring, Bot. Gaz. 29: 312, 1900.

Fig. 9-11

Colônia em BDA com crescimento lento, atingindo 4,0 cm diâm. aos sete dias de cultivo, velutina, zonada, cinza-oliváceo, tornando-se mais clara na margem; reverso zonado, cinza-escuro oliváceo, margem regular pardacenta; sinemas castanho-claros, 420-739x 11,5-14,5 $\mu \mathrm{m}$; setas estéreis subhialinas a castanhoclaras, não ramificadas, sinuosas, 74,8-203,6× 2,8-3,7 $\mu \mathrm{m}$; células conidiogênicas, 4,0-5,5×3,0-3,6 $\mu \mathrm{m}$; conídios catenulados, unicelulares, ovóides a mitriformes, lisos, castanho-claros, ápice arredondado, base truncada, 3,9-6,5×3,8-4,9 $\mu \mathrm{m}$.

Material examinado: BRASIL. Pernambuco: Recife, VI/1996, IX/1996, III/1997, IX/1997, XII/1997, água, Cavalcanti s.n. (Micoteca URM-3744).

Distribuição geográfica: cosmopolita (Domsch et al. 1993).

Comentários: Ellis (1971a) faz referência ao táxon isolado de Cola sp., Ipomoea sp., Lycopersicon sp., Saccharum sp., Solanum sp., Vicia sp., de solo, de papel e de material têxtil, na Europa, Egito, Índia, Iraque, Jamaica, Nigéria, América do Norte e Paquistão. Swart (1964) estudou o modo de formação do sinema em três espécies de Trichurus: T. gorgonifer Bain, T. terrophilus Swift \& Povah e T. spiralis. Morris (1963) e Ellis (1971a) indicam T. cylindricus Clements \& Shear como espécie-tipo do gênero, isolado do solo e de Cucurbita maxima Wright \& Marchand (1972) isolaram T. spiralis de solo, na Argentina.

\section{Agradecimentos}

Os autores agradecem às Professoras Leonor Costa Maia, Maria José Fernandes e Débora Massa Lima, do Departamento de Micologia da Universidade Federal de Pernambuco e aos revisores anônimos, pelas sugestões e críticas, e à CAPES, pelo suporte financeiro à primeira autora.

\section{Referências bibliográficas}

Abel, T.H. \& Barlocher, F. 1984. Effects of cadmium on aquatic Hyphomycetes. Applied and Environmental Microbiology 48: 245-251.

Agnihothrudu, V. 1963. Notes on fungi from norte-east India. XVI. Two rare Hyphomycetes from Assan. Sydowia 16(1-6): 141-143.

Alexopoulos, C.J.; Mims, C.W. \& Blackwell, M. 1996. Introductory Mycology. New York, John Wiley \& Sons.

Castañeda-Ruiz, R.F.; Iturriaga, T.; Minter, D.W.; Saikawa, M.; Vidal, G. \& Velázquez-Noa, S. 2003. Microfungi from Venezuela. A new species of Brachydesmiella, a new combination, and new records, Mycotaxon 85: 211 - 229.

Christensen, M.A. 1989. A view of fungal ecology. Mycologia 81(1): 1-19.

Clements, F.E. \& Shear, C.L. 1931. The genera of Fungi. New York, H.W. Wilson Co.

Crane, J.L. 1972. Illinois Fungi. III. Transactions of the British Mycological Society 58(3): 423-426.

Domsch, K.H.; Gams, W. \& Anderson, T. 1993. Compendium of Soil Fungi. London, Academic Press. v.1.

Ellis, M.B. 1971a. Dematiaceous Hyphomycetes. Kew, Commonwealth Mycological Institute.

Ellis, M.B. 1971b. Dematiaceous Hyphomycetes X. Mycological Papers 125: 1-31.

Field, J.I. \& Webster, J. 1983. Anaerobic survival of aquatic fungi. Transactions of the British Mycological Society 81(2): 365-369.

Fisher, P.J.; Davey, R.A. \& Webster, J. 1983. Degradation of lignin by aquatic and aero aquatic Hyphomycetes. Transactions of the British Mycological Society 80: 166-168.

Freire, F.C.O. 2005. An updated list of fungi from Ceará State (Brazil) - I Hyphomycetes. Revista Ciência Agronômica 36(3): 364-370.

Freire, F.C.O. \& Kozakiewicz, Z. 2005. Filamentous fungi, bacteria and yeasts associated with cashew kernels in Brazil. Revista Ciência Agronômica 36(2): 249-154.

Grandi, R.A.P. 1998. Hyphomycetes decompositores do folhedo de Alchornea triplinervia (Spreng.) Müll. Arg. Hoehnea 25(2): 133-148.

Grandi, R.A.P. 1999. Hifomicetos decompositores do folhedo de Euterpe edulis Mart. Hoehnea 26(1): 87-101.

Grandi, R.A.P. \& Attili, D.S. 1996. Hyphomycetes on Alchornea triplinervia (Spreng.) Müll. Arg. leaf litter from the Ecological Reserve Juréia-Itatins, State of São Paulo, Brazil. Mycotaxon 60: 373-386.

Grandi, R.A.P. \& Gusmão, L.F.P. 2002. Hyphomycetes decompositores do folhedo de Tibouchina pulchra Cogn. Revista Brasileira de Botânica 25(1): 79-87.

Gusmão, L.F.P. \& Grandi, R.A.P. 1997. Hyphomycetes com condioma dois tipos esporodóquio e sinema associados a folhas de Cedrela fissilis (Meliaceae), em Maringá, PR. Brasil. Acta Botanica Brasilica 11(2): 123-134.

Hasselbring, H. 1900. Comparative study of developmrnt of Trichurus spiralis and Stysanus stemonitis. Botanical Gazete 29: 312-369 
862 Cavalcanti \& Milanez: Hyphomycetes isolados da água e do solo, da Reserva Florestal de Dois Irmãos, Recife, PE...

Hoog, G.S. \& Guarro, J. 1995. Atlas of Clinical Fungi. Delft, Centraalbureau voor Schimmelcultures.

Hoog, G.S. \& Papendorf, M.C. 1976. The genus Phaeoisaria. Persoonia 8(4): 407-414.

Jain, B.L. 1962. Two new species of Curvularia. Transactions of the British Mycological Society 45: 539-544.

Kendrick, B. 1992. The Fifth Kingdom. Waterloo, Ontario, Mycologue Publ.

Machado, I.C.; Lopes, A.V. \& Porto, K.C. 1998. Reserva Ecológica de Dois Irmãos: Estudos em um remanescente de Mata Atlântica em área urbana (Recife - Pernambuco - Brasil). Recife, Editora Universitária, Universidade Federal de Pernambuco.

Maia, L.C. 1983. Sucessão de fungos em folhedo de floresta tropical úmida. Recife, Editora Universitária, Universidade Federal de Pernambuco.

Maia, L.C. 1998. Diversidade de fungos e líquens e sucessão fúngica na Reserva Ecológica de Dois Irmãos. Pp. 85-113. In: I.C. Machado; A.V. Lopes \&. K.C. Pôrto (eds.). Reserva Ecológica de Dois Irmãos: Estudos em um remanescente de Mata Atlântica em área urbana de (Recife, Pernambuco - Brasil). Recife, Editora Universitária, Universidade Federal de Pernambuco.

Maia, L.C.; Yano-Melo, A.M. \& Cavalcanti, M.Q. 2002. Diversidade de Fungos no Estado de Pernambuco. Pp. 15-50. In: M. Tabarelli \& J.M.C. Silva (eds.). Diagnóstico da Biodiversidade de Pernambuco. Recife, Secretaria de Ciências, Tecnologia e Meio Ambiente, Editora Massangana. v.1.

Martin, J.P. 1950. Use of rose bengal and streptomycin in the plate method for estimating soil fungi. Soil Science 69: 215-232.

Mazzanti de Castañòn, M.A. \& Gutiérrez de Arriola, S.A. 1995. Contribución al conocimento de la podredumbre castaño-rojiza de la vaina del arroz. Boletín Micológico 10(1-2): 47-52.

Moore-Landecker, E. 1996. Fundamentals of Fungi. 9th ed. New Jersey, Prentice-Hall.

Morris, E.F. 1963. The synnematous genera of the Fungi Imperfecti. Macomb, Western Illinois University, Series in Biological Sciences 3: 1-140.

Plakidas, A.G. \& Edgerton, C.W. 1936. A new imperfect fungi. Mycologia 28: 82-84.

Raghukumar, C. 2000. Fungi from marine habitats: an application in bioremediation. Mycological Research 104: $1222-1226$.
Remacle, J. 1981. The impact of fungi on environmental biogeochemistry. Pp. 73-87. In: D.T. Wicklow \& G.C. Carrol (eds.). The Fungal Community. New York, Marcel Dekker.

Rivera, M.N. 2003. Mycological survey of Río Camuy Caves Puerto Rico. Journal of Caves and Karst Studies 65(1): 23-28.

Schoenlein-Crusius, I.H. \& Milanez, A.I. 1998a. Fungos microscópicos da Mata Atlântica de Paranapiacaba, São Paulo, Brasil. Revista Brasileira de Botânica 21(1): 73-79.

Schoenlein-Crusius, I.H. \& Milanez, A.I. 1998b. Fungal succession on leaves of Alchornea triplinervia (Spreng.) Muell. Arg. submerged in a stream of an Atlantic Rainforest in the State of São Paulo, Brazil. Revista Brasileira de Botânica 21(3): 253-259.

Sivanesan, A. 1985. Graminicolous species of Bipolaris, Drechslera, Exserohilum and their teleomorphs. Transactions of the British Mycological Society 84(13): 548-551.

Sridhar, K.R. \& Kaveriappa, K.M. 1989. Observations on aquatic Hyphomycetes of the Westen Ghat streams, India. Nova Hedwigia 49(3-4): 455-467.

Swart, H.J. 1964. A study of the production of coremia in three species of the genus Trichurus. Antonie van Leeuwenhoek 30: 257-260.

Warcup, F.H. 1967. Fungi in Soil. Pp. 51-110. In: A. Burges \& F. Raw (eds.). Soil Biology. London, Academic Press.

Watanabe, T.; Katayama, S.; Enoki, M.; Honda, Y. \& Kuwahara, M. 2000. Formation of acyl radical in lipid peroxidation of linolenic acid by manganese-dependent peroxidase from Ceriporiospsis subvermispora and Bjerkandera adusta. European Journal Biochemistry 267: 4222-4231.

Wellbaum, C.; Schoenlein-Crusius, I.H. \& Santos, V.B. 1999. Fungos filamentosos em folhas do ambiente terrestre e aquático da Ilha dos Eucaliptos, Represa do Guarapiranga, São Paulo, SP. Revista Brasileira de Botânica 22(1): 69-74.

Wright, J.E. \& Marchand, S. 1972. Micoflora del suelo de la Argentina. III. Boletín de la Sociedad Argentina de Botánica 14: 305-310.

Wright, E.D.; Howlader, A.; Clayton.; Y.M.; Howlader, A.; Nazru, I. \& Husain, R. 1990. Keratomycosis caused by Dichotomophthropsis nymphaeearum. Mycoses 33(9-10): 477-481. 